\title{
Homogeneous nucleation of supercooled water: Results from a new equation of state
}

\author{
C. A. Jeffery and P. H. Austin \\ Atmospheric Sciences Programme, Department of Geography, University of British Columbia \\ Vancouver, British Columbia, Canada
}

\begin{abstract}
A series of laboratory and aircraft measurements have indicated that supercooled liquid water exists to temperatures as low as $-70^{\circ} \mathrm{C}$. These measurements also show that classical nucleation theory, using standard values for the thermodynamic properties of supercooled water, underestimates the nucleation rate of ice in liquid water at large supercoolings. New theoretical estimates for this homogeneous nucleation rate are presented, based on a new analytic equation of state for liquid water. The new equation of state, which is accurate over a pressure range of 3000 atmospheres and a temperature range of $1200 \mathrm{~K}$, is used to infer the latent heat of melting, liquid water density, and ice-water surface energy of supercooled water. Predictions of the nucleation rate and the homogeneous freezing temperature made by this equation of state are in agreement with observations at temperatures as cold as $-70^{\circ} \mathrm{C}$ and at pressures as high as 2000 atmospheres. These results indicate that it is not necessary to invoke a phase transition at $-45^{\circ} \mathrm{C}$ to explain aircraft and laboratory observations of homogeneous ice nucleation in supercooled water clouds.
\end{abstract}

\section{Introduction}

During the last 15 years several new measurements of the rate of homogeneous nucleation of ice in supercooled liquid water have become available. These data include nucleation rate measurements in an expansion cloud chamber to $-45^{\circ} \mathrm{C}$ [Hagen et al., 1981] and cirrus observations of supercooled water at $-35^{\circ} \rightarrow-40^{\circ} \mathrm{C}$ [Sassen and Dodd, 1988; Heymsfield and Miloshevich, 1993]. Nucleation rates reported by these investigators are substantially higher than those predicted by classical nucleation theory using standard estimates of the physical properties of water extrapolated to supercooled temperatures [Pruppacher, 1995].

The discrepancy between observed and calculated nucleation rates is one symptom of a larger problem: the absence of a physical model of liquid water that accounts for its unusual properties. These properties include the density maximum at $4^{\circ} \mathrm{C}$ (1 atmosphere) and the rapid change in the specific heat $\left(c_{p}\right)$ and other thermodynamic variables as liquid water is cooled to $T_{s}=-45^{\circ} \mathrm{C}$. Speedy and Angell [1976] and Speedy $[1982 \mathrm{a}, \mathrm{b}]$ proposed the "stability limit conjecture" to explain the apparently asymptotic behavior of the heat capacity, density, and latent heat, postulating a limit to the mechanical stability of liquid water at $T_{s}$ and atmospheric pressure.

Copyright 1997 by the American Geophysical Union.

Paper number 97JD02243.

0148-0227/97/97JD-02243\$09.00
Recently, Pruppacher [1995] used this idea to explain the failure of classical nucleation theory to predict observed nucleation rates at low temperatures. He extrapolated steep increases in the density, heat capacity, latent heat of evaporation, and viscosity and steep decreases in the density, ice-water surface energy, and latent heat of melting consistent with a second-order phase transition at $-45^{\circ} \mathrm{C}$. He then used these new values and the observed nucleation rate to derive the activation energy required to diffuse water molecules across the ice-water interface.

Although Pruppacher's approach was consistent with most measurements of water properties available prior to 1995, experiments conducted by Xie et al. [1993], Bartell and Huang [1994], and Huang and Bartell [1995] have cast fundamental doubt on the idea of a stability limit for liquid water at atmospheric temperatures and pressures. Xie et al. measured the absolute structure factor of liquid water to $-34^{\circ} \mathrm{C}$ and found no change in the correlation lengths of density fluctuations as $T_{s}$ was approached, in contrast to the strong fluctuations to be expected because of a stability limit. Huang and Bartell used electron diffraction measurements of a beam of water clusters condensing in supersonic flow to establish the presence of liquid water at $T=-70^{\circ} \mathrm{C}$ and to infer a nucleation rate at that temperature. Given the large viscosity of water at $-70^{\circ} \mathrm{C}$, it is possible that the liquid droplets observed in these experiments were nucleating at timescales longer than the microsecond observation times of the electron diffraction measurement. However, recent computer simulations indicate that equilibration 
times are of the order of a few nanoseconds, making it likely that the liquid phase is, indeed, still mechanically stable at these very low temperatures [Tanaka, 1996; Speedy, 1996].

In this paper we will use a new equation of state for water to derive homogeneous nucleation rates in the temperature range $-70^{\circ} \mathrm{C}<T<-30^{\circ} \mathrm{C}$ and the pressure range $1<p<2000$ atmospheres. Our approach is similar in spirit to Pruppacher's [1995], in that we reexamine classical nucleation theory in light of new information that has recently become available on the properties of liquid water. It differs from his in that an analytic equation of state, rather than extrapolations based on the stability limit conjecture, will provide the temperature and pressure dependence of the density, entropy, latent heat, and surface energy of liquid water.

The activation energy for diffusion across the icewater interface is also needed for the nucleation rate and cannot be obtained directly from the equation of state; this quantity will be estimated independently using recent measurements of the self-diffusion of water. The resulting pressure and temperature dependence of the nucleation rate calculated from these parameters (and a derived quantity called the homogeneous freezing temperature) are in agreement with the laboratory and aircraft measurements mentioned above, including the new $T=-70^{\circ} \mathrm{C}$ nucleation results of Huang and Bartell [1995].

We briefly review the classical nucleation equation and present the equation of state in section 2 . In sections 3,4 , and 5 we estimate the ice-water interface energy, the energy of germ formation, and the activation energy. We show the temperature and pressure dependence of the nucleation rate and homogeneous freezing temperature calculated from these energies in section 6 and summarize the results in section 7 .

\section{Equation of State}

The classical rate of homogeneous nucleation of an ice germ in a liquid water droplet is given by

$$
\begin{aligned}
J= & \left\{2 N_{c}\left(\frac{\rho_{w} k T}{\rho_{i} h}\right)\left(\frac{\sigma_{i / w}}{k T}\right)^{1 / 2} \exp \left[-\frac{\Delta g^{\ddagger}}{k T}\right]\right\} \times \\
& \exp \left[-\frac{\Delta F_{g}}{k T}\right]
\end{aligned}
$$

where $N_{c}$ is the number of monomers of water in contact with unit area of the ice surface, $\rho_{w}$ is the liquid water density, $\rho_{i}$ is the density of ice, $T$ is the temperature and $N_{c}$ can be estimated accurately as $5.85 \times 10^{14} \mathrm{~cm}^{-2}$ [Pruppacher and Klett, 1997, equation 7-51] (see the notation list for a complete list of symbols). The term in (1) enclosed in braces (the nucleation prefactor) represents the diffusive molecular flux across the liquidsolid interface. Recently, Oxtoby and Harrowell [1992] have argued that the prefactor used in (1) underestim- ates the molecular jump rate across the interface. Alternative formulations for the prefactor change the nucleation rate by 1-2 orders of magnitude at temperatures above $200 \mathrm{~K}$ [see, e.g., Huang and Bartell, 1995, Figure 7].

Evaluation of (1) requires estimates of the densities $\rho_{i}$ and $\rho_{w}$, the free energy of germ formation $\Delta F_{g}$, the activation energy $\Delta g^{\neq}$, and the interface energy $\sigma_{i / w}$. The energy of formation $\Delta F_{g}$, in turn, requires the surface energy $\sigma_{i / w}$ and an estimate of the equilibrium germ radius [Pruppacher and Klett, 1997, equation 7-52, with correction]:

$$
\Delta F_{g}=\frac{4 \sigma_{w / i} \pi a_{g}^{2}}{3} .
$$

Determination of $a_{g}$ is discussed in Section 4; it also requires knowledge of the liquid water density $\rho_{w}$ and the latent heat of melting $L_{m}$.

We use standard values for the ice density $\rho_{i}[$ Pruppacher and Klett, 1997, equation 3-2] and in section 3 derive a relation between $L_{m}$ and the interface energy $\sigma_{i / w}$. The density $\rho_{w}$ can be found from an equation of state of the form $\rho_{w}=f(p, T)$, which also yields $L_{m}$ using Maxwell's relations:

$$
\begin{aligned}
p & =-\left(\frac{\partial a}{\partial \alpha_{w}}\right)_{T}, \\
s_{w} & =-\left(\frac{\partial a}{\partial T}\right)_{\alpha_{w}}, \\
L_{m} & =T\left(s_{w}-s_{i}\right),
\end{aligned}
$$

where $p$ is the pressure, $a$ is the specific Helmoltz free energy, $\mathbf{s}$ is the specific entropy, $\alpha_{w}$ is the specific volume $\left(1 / \rho_{w}\right)$, and the subscripts $w$ and $i$ denote liquid and ice, respectively. The entropy of ice $s_{i}$ is assumed to be independent of pressure; it is determined from an analytic integration of the heat capacity of ice [Pruppacher and Klett, 1997, equation 3-12]:

$$
s_{i}=1.885 \log (T)+0.132 T-5.115
$$

where $\mathrm{T}$ is in Kelvins and $s_{i}$ is in $J k^{-1} K^{-1}$.

The analytic equation of state used here is described in detail by Jeffery [1996] and will be treated in this paper as an empirical relation. We include a brief outline of the derivation in Appendix A, with numerical values of the fitted parameters; in this section we show densities and heat capacities. The equation of state has the following form:

$$
p=F\left(\rho_{w}, T\right)=p_{0}+2 p_{h b},
$$

where $F$ is a function discussed in Appendix A. The factor of 2 in (5) reflects the 2 moles of hydrogen bonds in each mole of water. Equation (5) is a "mixture model"; the hydrogen bonds act as a separate species of water, exerting their own pressure $p_{h b}$. The hydrogen bond fraction is temperature dependent; its form is also 
given in Appendix A. Above freezing the bond fraction falls to zero, and the only contribution to the pressure comes from the background pressure term $p_{0}$. By considering separately the effect of hydrogen bonds on the free energy $a$ and (through (3a)) the pressure p, (5) is able to accurately predict the thermodynamic properties of water at supercooled temperatures.

The mixture model described here was first employed by Poole et al. [1994], who used the Van der Waals equation to calculate $p_{0}$ and demonstrated qualitatively that (5) was able to produce the density maximum of water. We have introduced a much more accurate form for $p_{0}$ and modified the $p_{h b}$ formulation of Poole et al. so that its influence is limited to supercooled temperatures.

Figures 1a and $1 \mathrm{~b}$ show the agreement between steam table [Haar et al., 1984] and supercooled measurements [Hare and Sorensen, 1987] and the equation of state in the pressure range 1 bar $<p<2800$ bars and the temperature range $-40^{\circ} \mathrm{C}<T<1000^{\circ} \mathrm{C}$. The equation predicts a density maximum at pressures up to 800 bars, as open hydrogen bonds force a local density related to their perfect tetrahedral geometry. The bonds are broken at pressures above 800 atmospheres, removing the density maximum. The displacement of the density maximum to lower temperatures with increasing pressure is in agreement with measurements [Angell, 1982, page 27].

We can compare the steam table values for the density with the present equation of state and three other cubic equations of state by grouping ( $\left.\mathrm{T}, \mathrm{p}, \rho_{w}\right)$ triplets in bins of width $24 \mathrm{~kg} \mathrm{~m}^{-3}$ in the temperature and pressure range $0^{\circ} \mathrm{C}<T<700^{\circ} \mathrm{C}, 0.1$ bar $<p<1200$ bars and calculating the average rms percentage deviation of

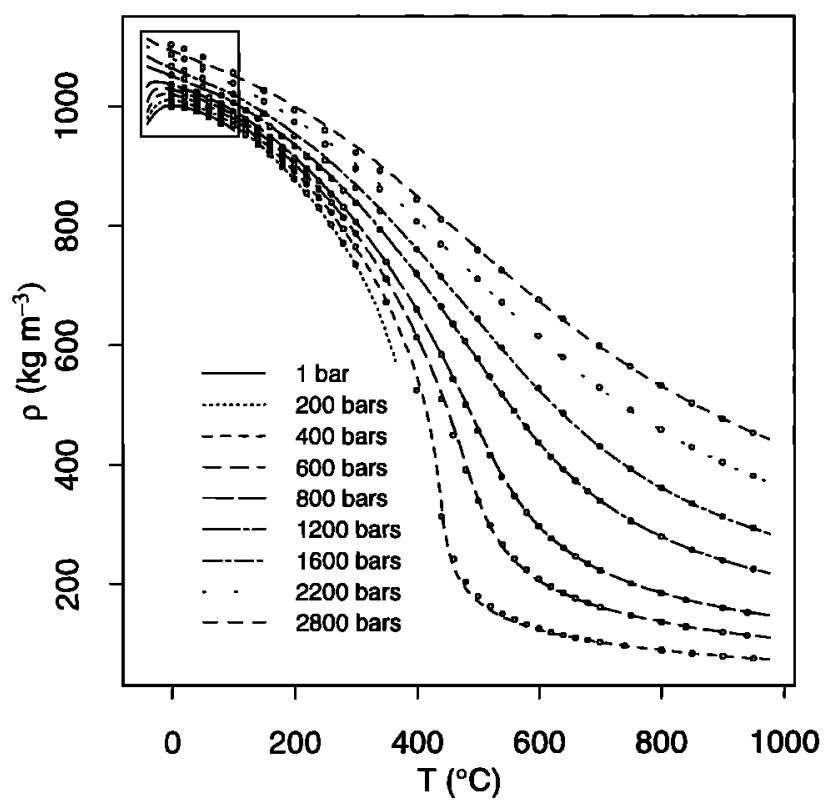

Figure 1a. Isobaric density of liquid water. Circle points are data from Haar et al. [1984] and Hare and Sorensen [1987]. Boxed region is expanded in Figure 1b.

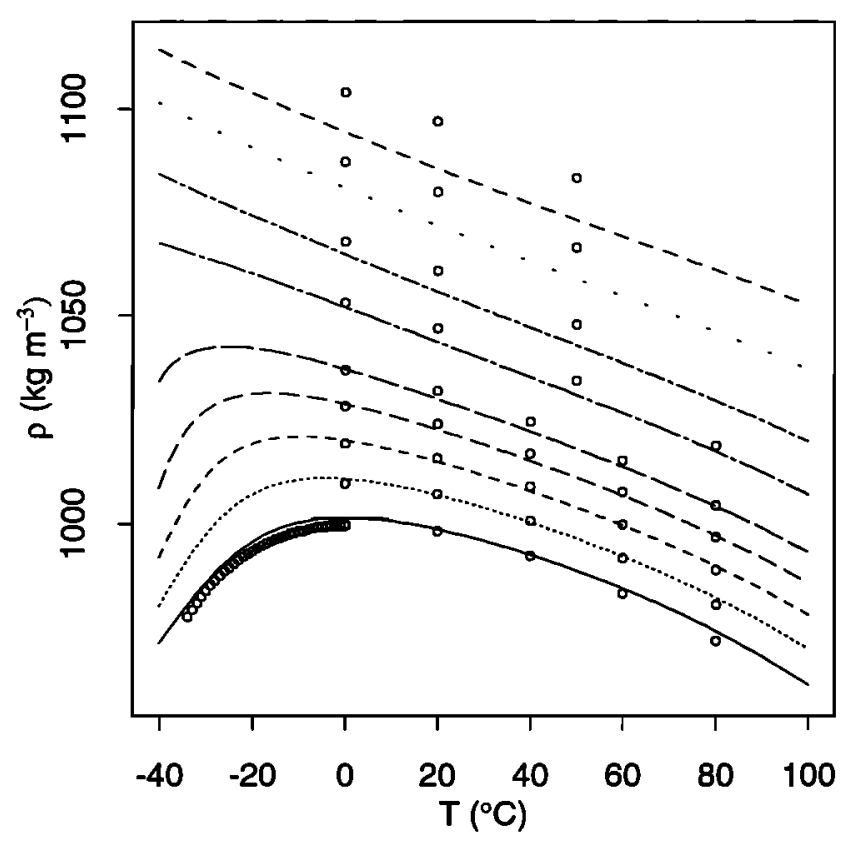

Figure 1b. As in Figure 1a but magnified for the temperature range $-40^{\circ} \mathrm{C}<T<100^{\circ} \mathrm{C}$.

the predicted density from the steam table values. The average rms percentage density deviation with this binning is $0.490,11.06,21.7$ and 59.7 for the present equation of state, Song and Mason [1989], Peng-Robinson [Melhem et al., 1989], and Van der Waals, respectively. Above supercooled temperatures the new equation of state is approximately 20 times more accurate than other equations of state of similar complexity. Adding supercooled data to the fit and extending the temperature and pressure range to $-34^{\circ} \mathrm{C}<T<1200^{\circ} \mathrm{C}$, and 0.1 bar $<p<3000$ bars decreases the average density deviation of the present equation of state from $0.49 \%$ to $0.41 \%$.

Figure 2 shows the heat capacity derived from (5) using (3a), (3b), and the Maxwell relation:

$$
c_{p}=T\left(\frac{\partial s_{w}}{\partial T}\right)_{T}
$$

At a pressure of 1 bar the equation of state matches the observed increase of $c_{p}$ as the temperature decreases to $-45^{\circ} \mathrm{C}$. The equation of state does not show a stability limit at supercooled temperatures. Rather there is a continuous change from the properties of supercooled water to those of ice. Not shown here are other water properties predicted by the the equation of state, such as the observed anomalous increase in $c_{p}$ at the critical point. The equation also predicts a phase transition between high-density and low-density liquid water above 1000 bars at supercooled temperatures and a second critical point for liquid water. These high pressure/low temperature results are discussed by Jeffery [1996]. 


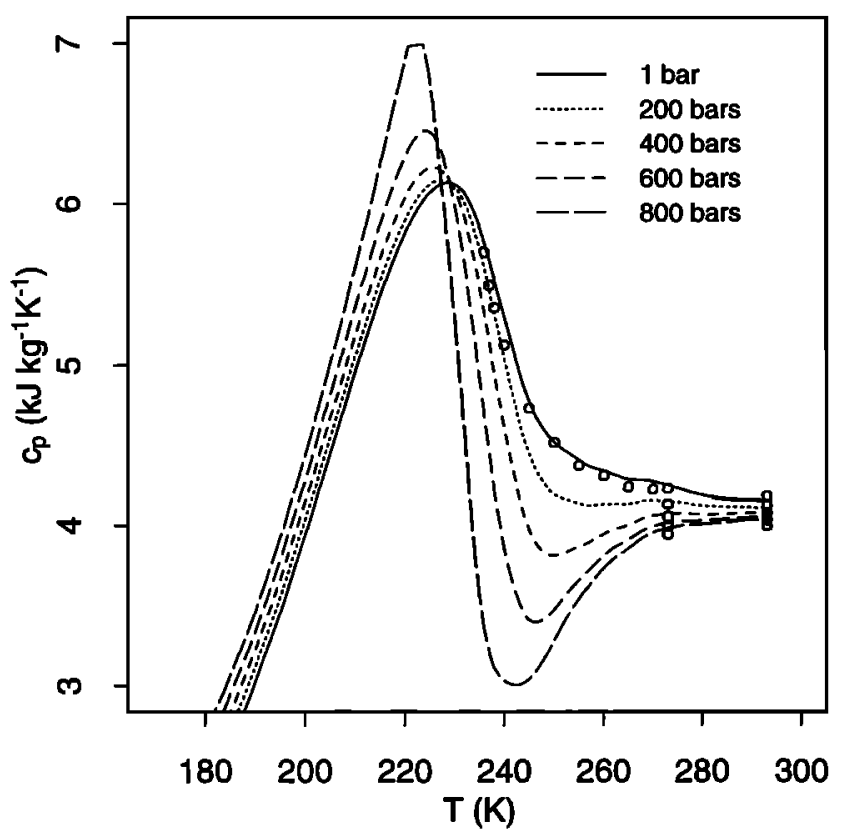

Figure 2. Isobaric heat capacity predicted by the new equation of state at five pressures. Circle points are data from Haar et al. [1984] and Angell et al. [1982].

\section{Ice-Water Surface Energy}

The equation of state provides the density $\rho_{w}$ and entropy $s_{w}$ of liquid water over a wide range of temperatures and pressure. The evaluation of the nucleation rate also requires the ice-water surface energy $\sigma_{i / w}$ as a function of temperature and pressure. We adopt a pressure-dependent empirical relation that relates $\sigma_{i / w}$ to the latent heat of melting $L_{m}$, which is predicted from the equation of state through (3c). The basis for this relation is the observation of Turnbull [1950], who noted that the solid-liquid interface energies $\sigma_{s l}$ of a number of metals and metalloids were closely related to $L_{m}$ through

$$
\sigma_{s l} \approx k_{T} L_{m} \rho_{s}^{2 / 3} / N_{A}^{1 / 3},
$$

where $\rho_{s}$ is the density of the solid phase in $\mathrm{mol} \mathrm{m}^{-3}$, $L_{m}$ is in $\mathrm{J} \mathrm{mol}{ }^{-1}$, and $N_{A}$ is Avogadro's number. The coefficient $k_{T}$ is about 0.45 for metals and 0.32 for water and several metalloids. A summary of Turnbull's results for materials with $k_{T} \approx 0.32$ is shown in Table 1 . We will use $k_{T}=0.32$ below.

Equation (7) cannot be derived from thermodynamics, but it has consistently been predicted by various molecular theories including, most recently, the inhomogeneous cell model of Liu [1993] and the density functional theory of McMullen and Oxtoby [1988].

As it stands, (7) does not capture the commonly assumed temperature dependence of $\sigma_{i / w}$ at atmospheric pressures. Equation (7) produces a $\sigma_{i / w}$ estimate that decreases significantly faster than typical estimates of the water-air surface energy or the ice-water surface energy [Floriano and Angell, 1990]. Dufour and Defay
Table 1. The $k_{T}$ Values for Different Metalloids

\begin{tabular}{lclc}
\hline Metal & $k_{T}$ & Metal & $k_{T}$ \\
\hline Water & 0.32 & Bismuth & 0.33 \\
Antimony & $\mathbf{0 . 3 0 2}$ & Germanium & 0.348 \\
\hline
\end{tabular}

From Turnbull [1950].

[1963, equation 13.95] and Pruppacher and Klett [1978, equation 5-47a] have produced temperature-dependent estimates of $\sigma_{i / w}$ based on scaling arguments using the latent heat of ice and water. Figure 3 shows these extrapolations of $\sigma_{i / w}$ together with the existing experimental data at $0^{\circ} \mathrm{C}$.

We will assume a similar temperature dependence for (7) using an empirically determined constant $k_{\sigma}$ :

$$
\sigma_{i / w}=k_{T} \mathcal{L}_{m} \rho_{i}^{2 / 3} / N_{A}^{1 / 3}-k_{\sigma} T
$$

where $\rho_{i}$ is the density of ice, $\mathrm{T}$ is in degrees Celsius, and $k_{\sigma}=0.00009 \mathrm{~J} \mathrm{~m}^{-2} \mathrm{~K}^{-1}$.

The numerical value of $k_{\sigma}$ is independent of temperature and pressure; it is chosen using (1) to yield the observed temperature dependence of the nucleation rate at 1 atmosphere in Section 6 . Figure 3 shows (8) evaluated at pressures of $1 \mathrm{bar}$, where it has approximately the same slope as Pruppacher and Klett's [1978] extrapolation, and at 550 bars, where the increase in $L_{m}$ with pressure has increased $\sigma_{i / w}$ at all temperatures.

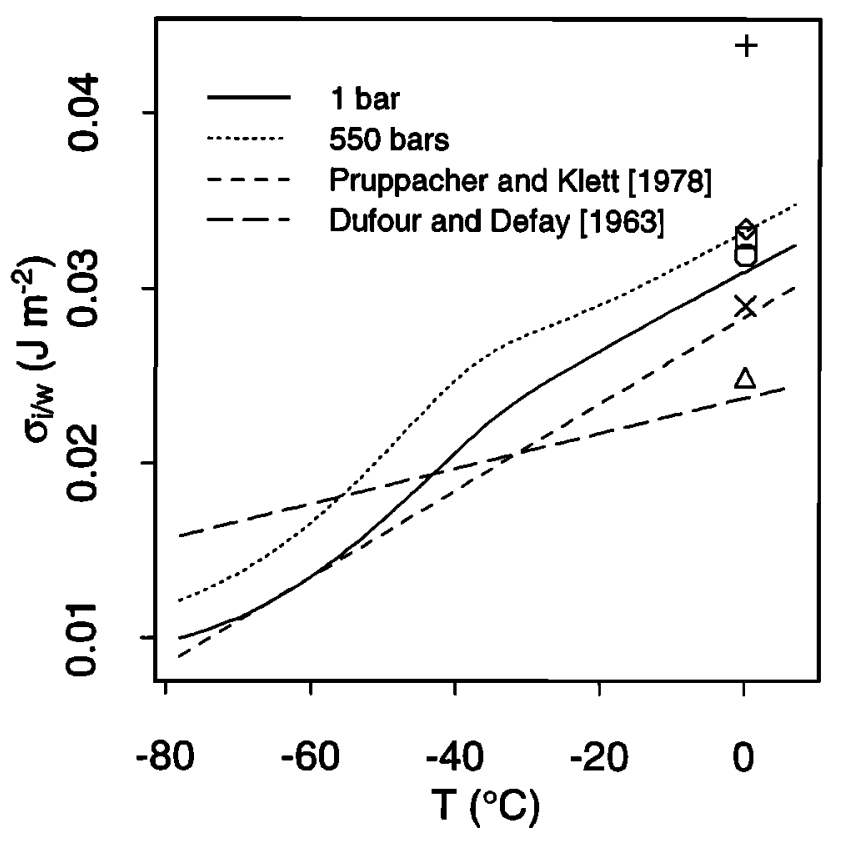

Figure 3. The value $\sigma_{i / w}$ at supercooled temperatures. Values at 1 bar and 550 bars are generated from (8) with $k_{T}=0.32$ and $k_{\sigma}=0.00009 \mathrm{~J} \mathrm{~m}^{-2} \mathrm{~K}^{-1}$. Curve labeled Pruppacher and Klett [1978] is calculated from their equation 5-47a and curve labeled Dufour and Defay [1963] is from their equation 13.95. Square is from Ketcham and Hobbs [1969], circle is from Wood and Walton [1970], triangle from Coriel et al. [1971], plus sign is from Jones [1973], cross is from Hardy [1977] and diamond is from Jones [1973]. 
No measurements are available to directly test the pressure dependence of (8). It can, however, be tested indirectly via predictions of the homogeneous freezing temperature. In section 6 we present homogeneous freezing temperature predictions made using (8) at pressures as high as 2000 atmospheres.

\section{Energy of Formation}

The free energy of germ formation $\left(\Delta F_{g}\right)$ defined by (2) is the energy required to construct an ice germ of critical radius $a_{g}$. The critical radius used in (2) is the equilibrium germ radius found by integrating the equilibrium condition for an ice germ in a supercooled droplet of pure water, which is itself in equilibrium with humid air. [Pruppacher and Klett, 1997, equations 6-52 and 6-10]:

$$
\begin{aligned}
\frac{L_{m}-\frac{2}{\rho_{\mathrm{s}}}\left(\frac{\sigma_{1 / w}}{a_{i}}\right)}{T} d T & +2\left(\frac{1}{\rho_{w}}-\frac{1}{\rho_{i}}\right) d\left(\frac{\sigma_{w / a}}{a_{d}}\right) \\
& -\frac{2}{\rho_{i}} d\left(\frac{\sigma_{i / w}}{a_{i}}\right)=0
\end{aligned}
$$

Here $a_{i}$ is the ice germ radius, and we have included in (9) a correction to the latent heat of fusion $L_{m}$ for the pressure difference between the germ and the surrounding liquid due to germ curvature. The surface energy $\sigma_{i / w}$ is estimated from the latent heat using (8).

We integrate (9) from the freezing point $\left(T_{0}, a_{i}=\infty\right)$ to an equilibrium temperature $T_{e}$ and equilibrium radius $a_{g}$ given values of $L_{m}$ from (3c), $\rho_{i}$ from Pruppacher and Klett [1997, equation 3-2] and $\sigma_{i / w}$ from (8). We show in Appendix B that to a good approximation (2) can be written as

$$
\Delta F_{g}=\frac{16 \pi \sigma_{i / w}^{3}}{3\left[\hat{L}_{m, c} \bar{\rho}_{i} \ln \left(T_{0} / T_{e}\right)\right]^{2}},
$$

where the averages $\widehat{L}_{m, c}$ and $\overline{\rho_{i}}$ are defined in Appendix B.

\section{Estimating the Activation Energy}

The ice-water activation energy $\Delta g^{\neq}$is the final physical quantity needed to evaluate the nucleation rate using (1). There are no direct measurements of $\Delta g^{F}$; it is typically inferred from two measurable quantities: the viscosity $\eta$ or the self-diffusivity $\mathrm{D}$. The defining relationships are [Glasstone et al., 1941]

$$
\begin{aligned}
D & =D_{0} \exp \left(-E_{D}(T) / R T\right) \\
\eta & =\eta_{0} \exp \left(E_{\eta}(T) / R T\right),
\end{aligned}
$$

where $D_{0}$ and $\eta_{0}$ are approximately independent of temperature. At temperatures near the melting point the energies in the Boltzman factor, $E_{D}$ and $E_{\eta}$, agree within experimental error and are about $4.6 \mathrm{Kcal} \mathrm{mol}^{-1}$
[Krynicki et al., 1980]. Thus $\Delta g^{\neq}$is equated to either $E_{D}\left[\right.$ Pruppacher, 1972] or to $E_{\eta}$ [Dufour and Defay, 1963]. We will determine $\Delta g^{\neq}$from $E_{D}(T)$ using the supercooled self-diffusivity measurements of Prielmeier et al. [1988]. These measurements were made on bulk samples of supercooled droplets at pressures as high as 3000 bars.

There are two different procedures used to calculate activation energies from self-diffusivity measurements; this has led to large discrepancies in the estimation of $\Delta g^{\ddagger}$. The most common procedure is to define the effective activation energy as [Pruppacher, 1972]:

$$
\Delta g^{\not}=E_{D}=-R d(\ln D) / d\left(T^{-1}\right) .
$$

This relationship can be derived directly from (11) or from thermodynamic arguments [Wang, 1952] and has the advantage of removing the unknown coefficient $D_{0}$ from the estimate of $\Delta g^{\neq}$. The implicit assumption in either the algebraic or thermodynamic derivation of (13) is that the temperature dependence of $E_{D}(T)$ is weak compared to the $1 / T$ dependence of the exponential. Although this condition is satisfied above $0^{\circ} \mathrm{C}$, it fails in the supercooled region where the temperature dependence of $\Delta g^{\neq}$is strong.

An alternative is to separately estimate both $D$ and $D_{0}$ and use (11) directly to determine $E_{D}$. The diffusivity $D(T)$ is available from Prielmeier et al. [1988], who have fit their self-diffusivity measurements in the temperature range $218 K<T<450 \mathrm{~K}$ and the pressure range 1 bar $<p<3000$ bars using an empirical Vogel-Tamman-Fulcher equation:

$$
D(T)=D_{*} \exp \left[-B /\left(T-T_{*}\right)\right] .
$$

Using (11), $\Delta g^{\neq}$is given by

$$
\Delta g^{\neq}=E_{D}=R T\left[\frac{B}{T-T_{*}}-\ln \left(\frac{D_{*}}{D_{0}}\right)\right] .
$$

To estimate $D_{0}$, we use a separate set of measurements by Harris and Woolf [1980] between $0^{\circ} \mathrm{C}$ and $50^{\circ} \mathrm{C}$ in the same pressure range. We assume that at these temperatures $E_{D}(T)$ is roughly constant and with this assumption fit (11) to the Harris and Woolf $D(T)$ values. The fit returns both $D_{0}$ and the average activation energy $\overline{E_{D}}$. Table 2 summarizes these results. The best fit average activation energy $\overline{E_{D}}$ is within the experimental error of $E_{D}\left(T=25^{\circ} \mathrm{C}\right)$ determined by Woolf [1975].

The activation energy predicted by (15) at various pressures is shown in Figure 4, which shows a significant decrease in $g^{\neq}$with increasing pressure. Four estimates of $\Delta g^{\neq}$at atmospheric pressure are shown in Figure 5. The solid line is a cubic fit [Pruppacher and Klett, 1978, equation 7-50a] derived using (13) and the self-diffusion data of Pruppacher [1972]. The shortdashed line labeled "Corrected" shows the estimate of $\Delta g^{\neq}$obtained when the data of Pruppacher [1972] is instead evaluated using (14) and (15). The long-dashed line labeled "Preilmeir [1988]" gives the $\Delta g^{\neq}$estimate 
Table 2. Self-Diffusivity Parameters for Equation (15)

\begin{tabular}{cccccc}
\hline$p$, bar & $D_{*}, 10^{10} \mathrm{~m}^{2} \mathrm{~s}^{-1}$ & $\mathrm{~B}, \mathrm{~K}^{-1}$ & $T_{*}, \mathrm{~K}$ & $D_{0}, 10^{10} \mathrm{~m}^{2} \mathrm{~s}^{-1}$ & $\overline{E_{D}}, \mathrm{~kJ} \mathrm{~mol}^{-1}$ \\
\hline 1 & 4.14 & 347 & 177 & 349 & 18.2 \\
100 & 6.46 & 455 & 161 & 328 & 18.0 \\
500 & 8.90 & 563 & 143 & 263 & 17.5 \\
1000 & 10.1 & 622 & 133 & 210 & 16.9 \\
1500 & 11.2 & 668 & 126 & 175 & 16.5 \\
2000 & 8.93 & 614 & 131 & 157 & 16.3 \\
\hline
\end{tabular}

$D_{*}, \mathrm{~B}$ and $T_{*}$ are from Prielmeier et al. [1988]. $D_{0}$ and $\overline{E_{D}}$ are from a fit to the results of Harris and Woolf [1980].

using (14) and (15) applied to the self-diffusion data of Prielmeier et al. [1988]. The dotted line labeled "Pruppacher [1995]" gives the correction proposed by Pruppacher [1995] to the usual $\Delta g^{\neq}$estimate obtained using (13).

Our analysis indicates that neglecting the $d E_{D} / d\left(T^{-1}\right)$ term in the derivation of (13) introduces a substantial error in the estimation of $E_{D}$ (and hence $\Delta g^{\ddagger}$ ) at supercooled temperatures. Pruppacher's [1995] correction to (13) (shown by the dotted line in Figure 5) assumes the formation of molecular clusters at low temperatures, which can increase transport of water molecules (and hence lower the ice-water activation energy) even as the activation energy for self-diffusion sharply increases [Pruppacher, 1995]. Although it is possible that molecular clusters do play a role in germ formation, the reanalysis presented in this section significantly reduces the motivation for introducing a speculative physical process into the formulation of $\Delta \boldsymbol{g}^{\neq}$. We will therefore use (15) and the usual assumption of equivalence between $\Delta g^{\neq}$and $E_{D}$ in our analysis of the nucleation equation in section 6 .

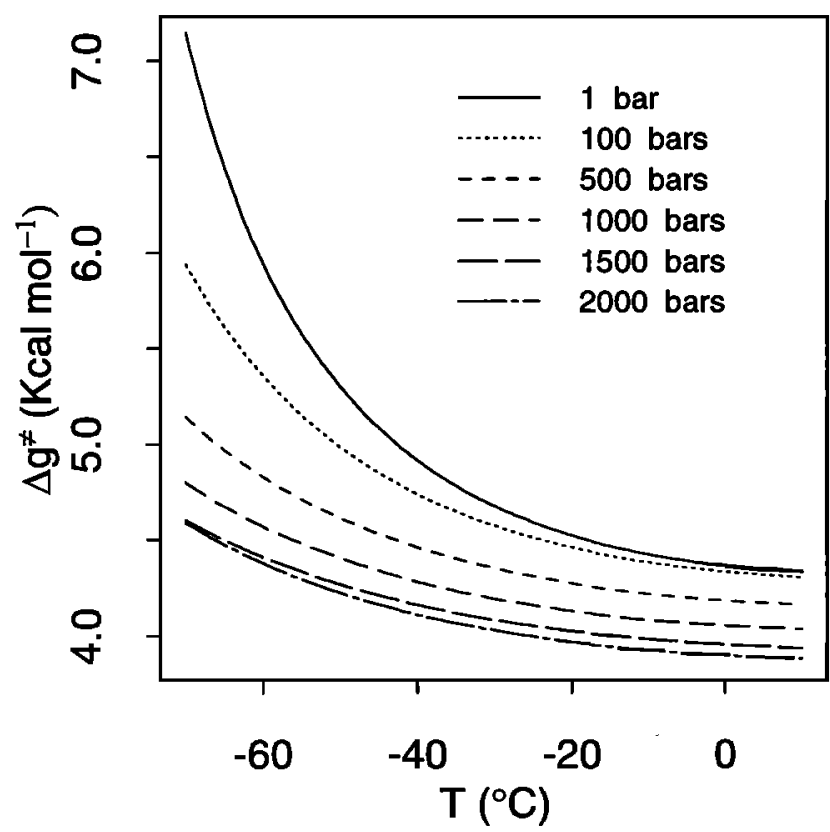

Figure 4. The value $\Delta g^{\neq}$at various pressures. Values calculated from equation (15) with parameter values from Table 2 .

\section{Homogeneous Nucleation Rate}

We are now in a position to evaluate (1) given the new values for $L_{m}, \sigma_{i / w}$, and $\Delta g^{\neq}$calculated from (3c), (8), and (15), respectively, with $\rho_{w}$ and $s_{w}$ given by the equation of state.

Figure 6: $0.83 * 5=4.15$ in Figure 6 shows the resulting nucleation rates, including the new nucleation measurement of Huang and Bartell [1995] using very small (3 $\mathrm{nm}$ radius) water droplets for the mother phase. These droplets have an internal pressure of $\approx 550$ bars; nucleation rates at this higher pressure are given by the dotted line. To evaluate the energy of formation $\left(\Delta F_{g}\right)$ for these small droplets, we use (B5) with $a_{d}=3 \mathrm{~nm}$ and $\sigma_{w / a}$ from Huang and Bartell [1995]:

$$
\sigma_{w / a}=111.63-0.13167 T
$$

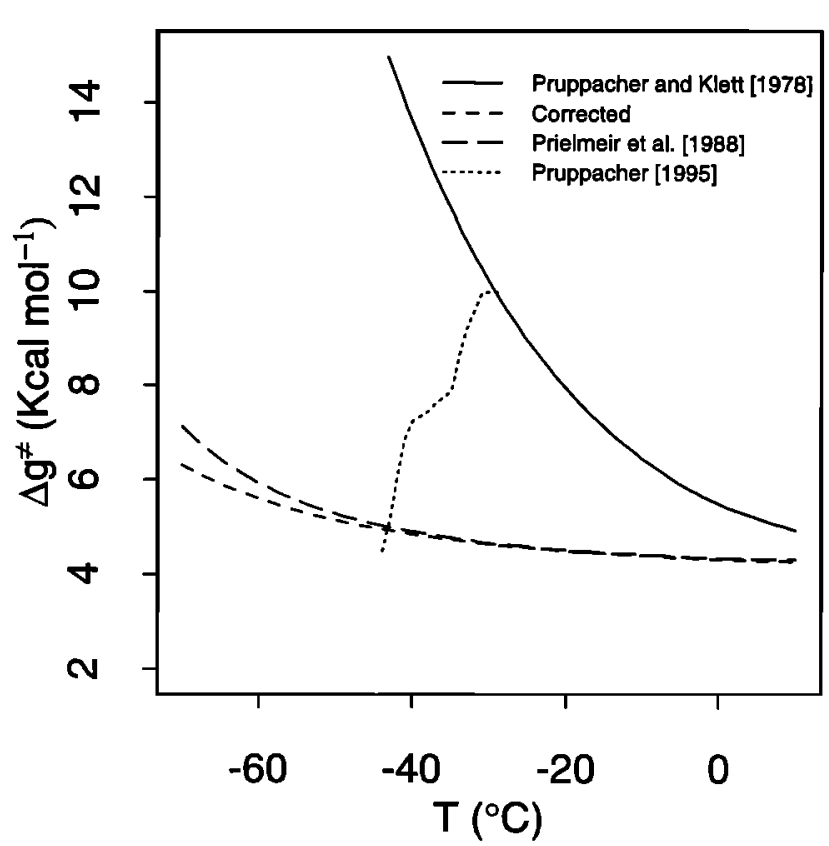

Figure 5. The value $\Delta g^{\ddagger}$ at supercooled temperatures. Line labels are "Pruppacher and Klett [1978]": Pruppacher's estimate using (13) and measurements from Pruppacher [1972]; "Corrected": a reanalysis of Pruppacher [1972] using (14) and (15) instead of (13); "Prielmeier et al. [1988]": values of $\Delta g^{\neq}$adopted here; and "Pruppacher [1995]", the correction to $\Delta g^{\neq}$used by Pruppacher [1995]. 


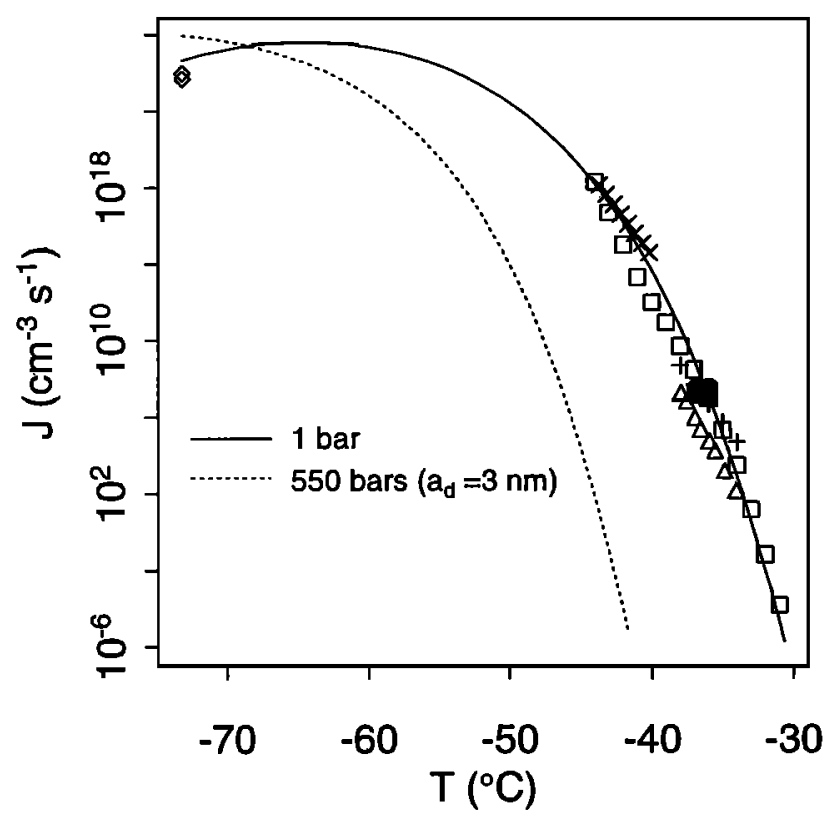

Figure 6. Comparison of $\mathrm{J}$ at 1 bar and 550 bars. The data of Huang and Bartell [1995] (diamonds) are at 550 bar because of the very small size of the droplets. The other data (listed below) are at 1 bar. Squares are from Pruppacher [1995], circles are from Wood and Walton [1970], triangles are from Butorin and Skripov [1972], plus signs are from Demott and Rogers [1990], and crosses are from Hagen et al. [1981].

where $\mathrm{T}$ is in Kelvins and $\sigma_{w / a}$ is in $\mathrm{mN} \mathrm{m}^{-1}$. The equilibrium melting temperature, $T_{M}$ was determined from Wagner et al. [1994]; $T_{M}$ is 0 at 1 bar, is -0.8 at 100 bars, is -4.1 at 500 bars, is -8.8 at 1000 bars, is -14.3 at 1500 bars, and is -21.0 at 2000 bars. The activation energy $\Delta g^{\neq}$was calculated at 500 bars, which introduces negligible error.

Figure 6 shows good agreement between observations at 1 atmosphere and the theoretical nucleation curve. The data and the theory are not completely independent, however, because the surface energy slope constant $\left(k_{\sigma}\right.$ in (8)) has been selected to produce the best fit to measurements at 1 atmosphere and temperatures warmer than $-45^{\circ} \mathrm{C}$. The Huang and Bartell [1995] data point at $-70^{\circ} \mathrm{C}$ and 550 bars provides one independent test of (8). Figure 6 also shows the effect of the strong pressure dependence in $\Delta g^{\neq}$on the nucleation rate. At one atmosphere the sharply increasing activation energy forces a decrease in $\mathrm{J}$ below $\mathrm{T}=-60^{\circ} \mathrm{C}$. At 550 atmospheres both the magnitude and the rate of increase of the activation energy are reduced (see Figure 4), and the decreasing $\sigma_{i / w}$ increases the nucleation rate as the temperature is reduced below $\mathrm{T}=-60^{\circ} \mathrm{C}$.

Additional tests are available from pressure-dependent measurements of the homogeneous freezing temperature $T_{H}$, which can be well-approximated as the temperature at which $99.99 \%$ of a population of droplets of volume $V_{d}$ will freeze homogeneously. Pruppacher and Klett [1997, equation 7-72] show that given the equilibrium melting temperature $T_{M}$, the cooling rate $\gamma_{c}$, and the droplet volume $V_{d}, T_{H} \approx T_{99.99}$ is given by

$$
\int_{T_{99.99}}^{T_{0}} J(T) d T=9.21 \frac{\gamma_{c}}{V_{d}}
$$

Figure 7 shows a comparison between (17) and observations in the pressure range 0.1 bar $<p<2000$ bars. The distance between opposing arrows indicates the range in $T_{H}$ produced by variations in droplet volume $V_{d}$ corresponding to radii between $0.1 \mu$ and $1000 \mu \mathrm{m}$. The nucleation rates capture the general downward trend in $T_{H}$ at pressures below 1000 bars, although the rate of change is underestimated. Included in Figure 7 are estimates of $T_{H}$ at 1500 and 2000 bars. These are connected by a dotted line on Figure 7, to emphasize their qualitative difference with the size-dependent $T_{H}$ values found at lower pressures. At pressures of 1500 bars and 2000 bars the equation of state predicts a phase transition at temperatures of $-73^{\circ} \mathrm{C}$ and $-93^{\circ} \mathrm{C}$, respectively. The phase transition (discussed in detail by Jeffery [1996]) between high-density and lowdensity liquid water rapidly forces droplet nucleation independent of droplet size.

\section{Summary and Conclusions}

We have used a new analytic equation of state to investigate the behavior of the classical homogeneous

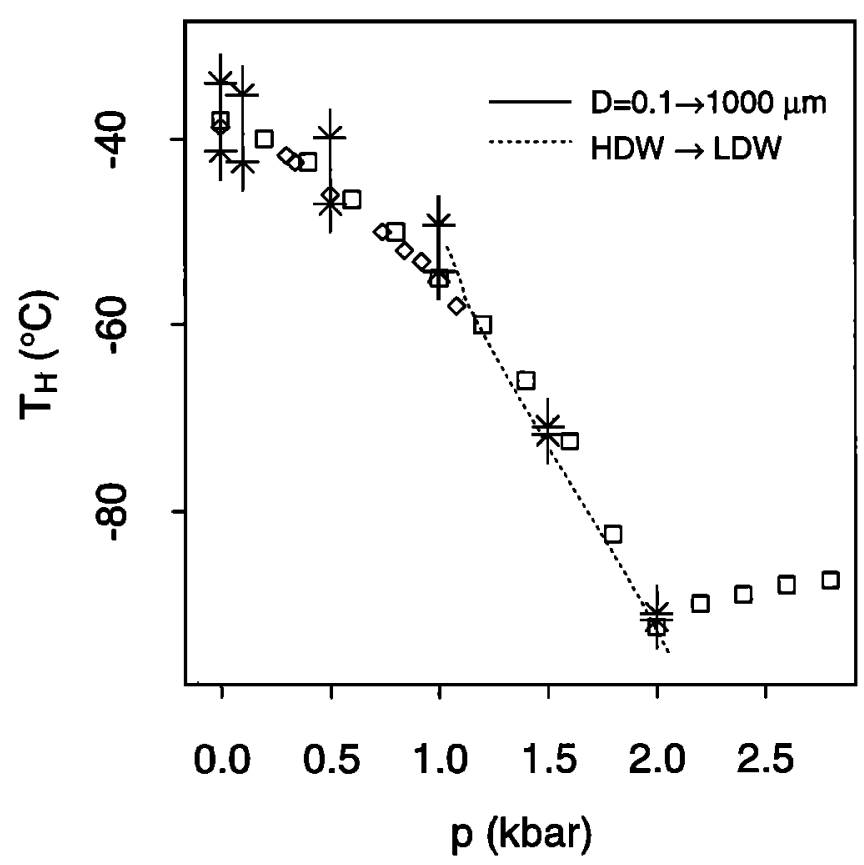

Figure 7. Effect of droplet diameter on $T_{H}$ at various pressures. The very short lines at 1500 and 2000 bars are a result of a HDW $\rightarrow$ LDW phase transition. The arrows positioned at the ends of the predicted $T_{H}$ values have been added for emphasis. Squares are from Kanno et al. [1975], and diamonds are from Xans and Barnaud [1975]. 
nucleation equation at supercooled temperatures and a range of pressures. The eight fitted coefficients listed in Appendix A are constrained by several thousand measurements of the temperature, pressure, density, and entropy of liquid water in the temperature range $-34^{\circ} \mathrm{C}<T<1200^{\circ} \mathrm{C}$ and the pressure range 1 bar $<p<3000$ bars. An additional constraint lies in the derivation of the equation of state, which is based on observations about the role played by strong hydrogen bonds in determining the properties of water [Jeffery, 1996; Jeffery and Austin, 1997, manuscript in preparation]. This foundation in a physical model for water substantially reduces the parameter space to be searched for the best nonlinear least squares fit to the data.

In this paper we have treated the equation of state as an empirical relationship and used it to infer the density and the latent heat of melting and through them the surface energy and free energy of germ formation. We have also presented an analysis of the activation energy for self-diffusion of water, suggesting that the temperature variation of the ice-water activation energy is less than is conventionally assumed at supercooled temperatures. In particular, our results indicate that laboratory and aircraft observations of homogeneous nucleation can be explained without the need to postulate abrupt changes in the activation energy at $T \approx-45^{\circ} \mathrm{C}$.

The parameter set, inserted into the classical nucleation equation, accurately predicts the recently observed nucleation rate of liquid water at $-70^{\circ} \mathrm{C}$ and atmospheric pressure, and the homogeneous freezing temperature at high pressures. We have followed many authors [e.g., Huang and Bartell, 1995; Pruppacher, 1995] in assuming that classical nucleation theory applies and that, for example, macroscopic parameters such as the solid-liquid surface energy $\sigma_{s l}$ have physical meaning for small water clusters away from the equilibrium freezing point. One example of an alternative approach, using an explicit partition function to calculate the free energy of formation $\Delta F_{g}$, was developed by Eadie Eadie [1971]. Future laboratory and aircraft measurements of the nucleation rate at temperatures between $-45^{\circ} \mathrm{C}$ and $-70^{\circ} \mathrm{C}$ and of the homogeneous freezing temperature with a variety of cooling rates and droplet sizes at high pressures would provide additional detailed tests of both classical and statistical-mechanical nucleation theory and of the predictions of the equation of state.

\section{Appendix A: Equation of State}

In this appendix we give the functional form of the equation of state. For a full discussion of the derivation, readers are referred to Jeffery [1996]. Programs (in Fortran77) that compare the density, entropy, and heat capacity of the equation of state against observations are available from the authors.

As discussed section 2, the equation has the following basic form:

$$
p=p_{0}+2 p_{H B} .
$$

For $p_{0}$ we use a Van der Waals-type equation that is a modified version of the Song and Mason [1990a, b] equation of state:

$$
\begin{aligned}
\frac{p_{0}}{\rho_{w} R T}=1 & +\left(b_{V W}-\frac{a_{V W}}{R T}\right) \rho_{w} \\
& +\alpha_{e o s} \rho_{w}\left[\frac{1}{1-\lambda b(T) \rho_{w}}-1\right] .
\end{aligned}
$$

Where $b(T)$ is related to the excluded volume and has the following functional form:

$$
\begin{aligned}
b(T) / v_{B} & =0.3 \exp \left(-12.15\left(T / T_{B}+0.1608\right)^{3}\right) \\
& -b_{1} \exp \left(1.066 T / T_{B}\right)+b_{2} .
\end{aligned}
$$

The second component of the fitted equation is the hydrogen bond term suggested by Poole et al. [1994]. We follow Poole et al. [1994] and write Helmholtz free energy as a simple partition function. We assume a combination of strong bonds with energy $\epsilon=\epsilon_{H B}$ and entropy $S=S_{H B}$ and weak bonds with energy $\epsilon=0$ and entropy $S=S_{0}$. The number of configurations of each bond type is then $\Omega_{0}=\exp \left(-S_{0} / R\right)$ and $\Omega_{H B}=$ $\exp \left(-S_{H B} / R\right)$, respectively.

The total hydrogen bond free energy is assumed to be a mixture of the two bond types [Jeffery, 1996]:

$$
\begin{aligned}
A_{H B}= & -f R T \ln \left[\Omega_{0}+\Omega_{H B} \exp \left(-\epsilon_{H B} / R T\right)\right] \\
& -(1-f) R T \ln \left(\Omega_{0}+\Omega_{H B}\right),
\end{aligned}
$$

where the mixture fraction $f$ is a function of temperature and density.

We separate the temperature and density dependence so that $f\left(T, \rho_{w}\right)=f^{*}\left(\rho_{w}\right) f^{* *}(T)$, where

$$
\begin{aligned}
f^{*}\left(\rho_{w}\right) & =\frac{1+C_{1}}{\exp \left[\left(\rho_{w}-\rho_{H B}\right) / \sigma\right]^{2}+C_{1}}, \\
f^{* *}(T) & =\exp \left[-0.18\left(T / T_{f}\right)^{8}\right] \\
\sigma & =C_{2} \rho_{H B},
\end{aligned}
$$

with $T_{f}=273.15 \mathrm{~K}$ and $0<C_{1}<1$.

Using (3a), we obtain for the pressure [Jeffery, 1996]

$$
\begin{aligned}
p_{H B}= & \rho_{w}^{2} \frac{\partial}{\partial \rho_{w}}\left(-f R T\left[\ln \Omega_{0}+\right.\right. \\
& \left.\left.\Omega_{H B} \exp \left(-\epsilon_{H B} / R T\right)-\ln \Omega_{0}+\Omega_{H B}\right]\right) \\
= & 2 f^{\prime} \rho_{w}^{2} \frac{\left(\rho_{w}-\rho_{H B}\right)}{\sigma^{2}} R T\left(\operatorname { l n } \left[\Omega_{0}+\right.\right. \\
& \left.\left.\Omega_{H B} \exp \left(-\epsilon_{H B} / R T\right)\right]-\ln \left[\Omega_{0}+\Omega_{H B}\right]\right),
\end{aligned}
$$

where

$$
\begin{aligned}
f^{\prime}= & \frac{\left(1+C_{1}\right) \exp \left[\left(\rho_{w}-\rho_{H B}\right) / \sigma\right]^{2}}{\left(\exp \left[\left(\rho_{w}-\rho_{H B}\right) / \sigma\right]^{2}+C_{1}\right)^{2}} \times \\
& \exp \left[-0.18\left(T / T_{f}\right)^{8}\right] .
\end{aligned}
$$


The entropy $s_{w}$ can now be obtained from (A1) using (3a) and (3b). The determination of the volumedependent function that results from the integration of (3b) is discussed in Jeffery [1996].

The following coefficients are returned from the fitting process (see the notation list for definitions):

$$
\begin{array}{ll}
\rho_{H B} & 0.8537 \mathrm{~g} \mathrm{~cm}^{-3} ; \\
S_{0} & -66.156 \mathrm{~J} \mathrm{~mol}^{-1} K^{-1}\left(\Omega_{0}=\exp \left(-S_{0} / R\right)\right) ; \\
S_{H B} & -4.464 \mathrm{~J} \mathrm{~mol}^{-1} K^{-1}\left(\Omega_{H B}=\exp \left(-S_{H B} / R\right)\right) ; \\
C_{1} & 0.3985 \\
C_{2} & 0.15758 \\
\alpha_{e o s} & 2.140 v_{B} \\
b_{1} & 0.24273 \\
b_{2} & 1.0298
\end{array}
$$

The following constants are passed to the fitting program and are not modified:

$$
\begin{array}{ll}
T_{B} & 1408.4 \mathrm{~K} ; \\
v_{B} & 4.1782 \times 10^{-5} \mathrm{~m}^{3} \mathrm{~mol}^{-1} ; \\
a_{V W} & 0.5542 \mathrm{~Pa} \mathrm{~m}^{6} \mathrm{~mol}^{-2} ; \\
b_{V W} & 4.44 \times 10^{-5} ; \\
\lambda & 0.30532 ; \\
\epsilon_{H B} & 13.5 \mathrm{~kJ} \mathrm{~mol}^{-1}
\end{array}
$$

\section{Appendix B: A Closed-Form Approximation to the Free Energy of Formation}

The derivation of the critical radius $a_{g}$ of an ice germ suspended in a drop of pure water of radius $a_{d}$, begins with (9), which relates the differentials of $\mathrm{T}, \boldsymbol{\sigma}, \boldsymbol{a}_{d}$ and $a_{i}$ at thermodynamic equilibrium. Integration of (9) from equilibrium at the freezing point $\left(T_{0}, a_{i}=\infty\right)$ to $\left(a_{g}, T_{e}\right)$ provides the equilibrium radius. In this appendix we will do this first by neglecting the curvature adjustment to the latent heat and the size dependence of the liquid drop (i.e., $\rho_{i} L_{m} \gg 2\left(\sigma_{i / w} / a_{i}\right)$ and $\left.d\left(\sigma_{i / w} / a_{i}\right) \gg d\left(\sigma_{w / a} / a_{d}\right)\right)$, then adding these terms. With both approximations we have the following from (9):

$$
-\int_{T=T_{0}}^{T_{e}} \frac{L_{m}}{T} d T-\int_{a_{\imath}=\infty}^{a_{\imath}} \int_{\sigma_{\imath} / w}^{\sigma_{\imath} / w\left(T_{0}\right)} \frac{2}{\rho_{i}} d\left(\frac{\sigma_{i / w}}{a_{i}}\right)=0 .
$$

Integrating (B1) gives

$$
\ln \frac{T_{0}}{T_{e}}=\frac{2 \sigma_{i / w}}{\widehat{L}_{m} \bar{\rho}_{i} a_{i}}
$$

where

$$
\widehat{L}_{m}=\frac{\int_{T=T_{0}}^{T_{e}} L_{m} / T d T}{\int_{T=T_{0}}^{T_{e}} 1 / T d T} \approx \frac{\overline{L_{m} / T}}{\overline{1 / T}}
$$

Note that because of the very weak temperature dependence of $\rho_{i}$, it can be separated out of the average $1 /\left(\rho_{i} T\right)$ and represented by a simple temperature average $\bar{\rho}_{i}$.

Substituting for $\frac{2}{\rho_{\iota}}\left(\frac{\sigma_{\imath / w}}{a_{\iota}}\right)$ in (2) using (B2) gives the corrected latent heat:

$$
\widehat{L}_{m, c}=\widehat{L}_{m}-\ln \left(T_{0} / T_{e}\right) \widehat{L}_{m} .
$$

Incorporating (B4) into (B2) and using (2) gives (10) in section 4 :

$$
\Delta F_{g}^{(1)}=\frac{16 \pi \sigma_{i / w}^{3}}{3\left[\widehat{L}_{m, c} \bar{\rho}_{i} \ln \left(T_{0} / T_{e}\right)\right]^{2}}
$$

If we also remove the large droplet approximation and include $\left(\frac{\sigma_{w / a}}{a_{d}}\right)$ in (9), (10) becomes

$$
\Delta F_{g}^{(2)}=\frac{16 \pi \sigma_{i / w}^{3}}{3\left[\hat{L}_{m, c} \bar{\rho}_{i} \ln \left(T_{0} / T_{e}\right)-w^{\prime}\right]^{2}},
$$

where

$$
\begin{aligned}
w^{\prime} & =\frac{2 \sigma_{w / a}}{a_{d} \bar{\rho}_{w}}\left(\hat{\rho}_{w}-\bar{\rho}_{i}\right), \\
1 / \hat{\rho}_{w} & =\frac{\int_{T=T_{0}}^{T_{e}} 1 / \rho_{w} d T}{\int_{T=T_{0}}^{T_{e}} d T} \approx \frac{\overline{1 / \rho_{w}}}{\bar{T}}
\end{aligned}
$$

and $\rho_{w}$ is the density of water.

We have compared values for $\Delta F_{g}^{(1)}$ and $\Delta F_{g}^{(2)}$ (using $a_{d}=1 \mu \mathrm{m}$ ) with a numerical integration of (9) between $-70^{\circ} \mathrm{C}<T<-30^{\circ} \mathrm{C}$ at atmospheric pressure. The rms deviations between the analytic and numeric values are $6.72 \times 10^{-21} \mathrm{~J}$ for $\Delta F_{g}^{(1)}$ and $4.41 \times 10^{-21} \mathrm{~J}$ for $\Delta F_{g}^{(2)}$. At these temperatures $\Delta F_{g}$ varies from 0 to $3.5 \times 10^{-19} \mathrm{~J}$, so that the standard deviations are 3 $4 \%$ of typical values. Therefore (10) and (B5) have been used here for calculations for $\Delta F_{g}$. The small difference between the analytic and numeric results also demonstrates that the effect of droplet curvature on $\Delta F_{g}$ is negligible for typical droplet sizes.

\section{Notation}

$\begin{aligned} \alpha_{e o s} & \text { temperature dependent function. } \\ \alpha_{w} & \text { specific volume of water (A2). } \\ \epsilon_{H B} & \text { energy of strong hydrogen bonds in water }\end{aligned}$ (A4).

$\eta \quad$ viscosity (12).

$\eta_{0} \quad$ viscosity coefficient (12).

$\lambda$ constant (A2).

$\rho_{H B} \quad$ hydrogen bond density (A5a).

$\rho_{i} \quad$ density of ice. 


\begin{tabular}{|c|c|}
\hline$\rho_{w}$ & density of water (1). \\
\hline$\sigma$ & $\mathrm{f}$ function width defined by (A5c). \\
\hline$\sigma_{i / w}$ & ice-water surface energy (8), \\
\hline$\sigma_{s l}$ & solid-liquid surface energy (7). \\
\hline$\sigma_{w / a}$ & water-air surface energy (16). \\
\hline$v_{B}$ & Boyle volume (A3). \\
\hline$\Omega_{0}$ & $\begin{array}{l}\text { number of configurations of weak hydro- } \\
\text { gen bonds (A4). }\end{array}$ \\
\hline$\Omega_{H B}$ & $\begin{array}{l}\text { number of configurations of strong hydro- } \\
\text { gen bonds (A4). }\end{array}$ \\
\hline$a$ & specific Helmholtz free energy (3b). \\
\hline$a_{d}$ & the radius of a water droplet (9). \\
\hline$a_{i}$ & the radius of an ice germ (9). \\
\hline$a_{V W}$ & Van der Waals constant (A2). \\
\hline$A_{H B}$ & $\begin{array}{l}\text { molar free energy of open tetrahedral hy- } \\
\text { drogen bonds (A4). }\end{array}$ \\
\hline$b$ & temperature dependent function (A3). \\
\hline$b_{1}, b_{2}$ & $\begin{array}{l}\text { fitted constants in the expression for b } \\
\text { (A3). }\end{array}$ \\
\hline$B$ & fitted constant defined by (14). \\
\hline$b_{V W}$ & Van der Waals constant (A2). \\
\hline & specific heat capacity. \\
\hline$C_{1}, C_{2}$ & $\begin{array}{l}\text { fitted constants in the expressions for } f\left(T, \rho_{w}\right) \\
\text { (A5a). }\end{array}$ \\
\hline$D$ & self-diffusivity (11) \\
\hline$D_{0}$ & self-diffusivity coefficient (11). \\
\hline$D_{*}$ & a fitted constant defined by (14). \\
\hline$E_{\eta}, E_{D}$ & $\begin{array}{l}\text { activation energies for viscosity and self- } \\
\text { diffusion (11) and (12). }\end{array}$ \\
\hline$f$ & $\begin{array}{l}\text { fraction of hydrogen bonds that are cap- } \\
\text { able of forming strong bonds. }\end{array}$ \\
\hline$f^{*}\left(\rho_{w}\right)$ & function defined by (A5a). \\
\hline$f^{* *}(T)$ & function defined by (A5b). \\
\hline$\Delta F_{g}$ & $\begin{array}{l}\text { free energy of formation of an ice germ } \\
(2) \text {. }\end{array}$ \\
\hline$\Delta g^{\neq}$ & ice-water activation energy (13). \\
\hline$h$ & Planck's constant. \\
\hline$J$ & homogeneous nucleation rate (1). \\
\hline$k$ & Boltzmann's constant. \\
\hline$k_{\sigma}$ & $\begin{array}{l}0.00009 \mathrm{~J} \mathrm{~m}^{-2} \mathrm{~K}^{-1} \text { slope constant in the } \\
\sigma_{i / w} \text { expression }(8) .\end{array}$ \\
\hline $\begin{array}{c}k_{T} \\
L_{m}\end{array}$ & $\begin{array}{l}0.32 \text {, an estimated constant defined in }(7) \text {. } \\
\text { latent heat of melting }(3 \mathrm{c}) \text {. }\end{array}$ \\
\hline$\widehat{L}_{m}$ & average latent (B3). \\
\hline$L_{m, c}$ & corrected latent heat including curvature. \\
\hline$N_{A}$ & Avogadro's number. \\
\hline$N_{C}$ & $\begin{array}{l}5.85 \times 10^{14} \mathrm{~cm}^{-2} \text {, the number water monomers } \\
\text { in contact with the ice surface. }\end{array}$ \\
\hline$p$ & pressure. \\
\hline$p_{0}$ & $\begin{array}{l}\text { background pressure from the new equa- } \\
\text { tion of state (A2). }\end{array}$ \\
\hline$p_{H B}$ & $\begin{array}{l}\text { pressure exerted by strong hydrogen bonds } \\
\text { (A6). }\end{array}$ \\
\hline $\begin{array}{r}s_{i}, s_{w} \\
R\end{array}$ & $\begin{array}{l}\text { specific entropy of ice and water. } \\
\text { universal gas constant. }\end{array}$ \\
\hline$S_{0}$ & weak bond entropy $\left(\Omega_{0}=\exp \left(-S_{0} / R\right)\right)$ \\
\hline $\begin{array}{r}S_{H B} \\
T\end{array}$ & $\begin{array}{l}\text { strong bond entropy }\left(\Omega_{H B}=\exp \left(-S_{H B} / R\right)\right) \text {. } \\
\text { temperature. }\end{array}$ \\
\hline
\end{tabular}

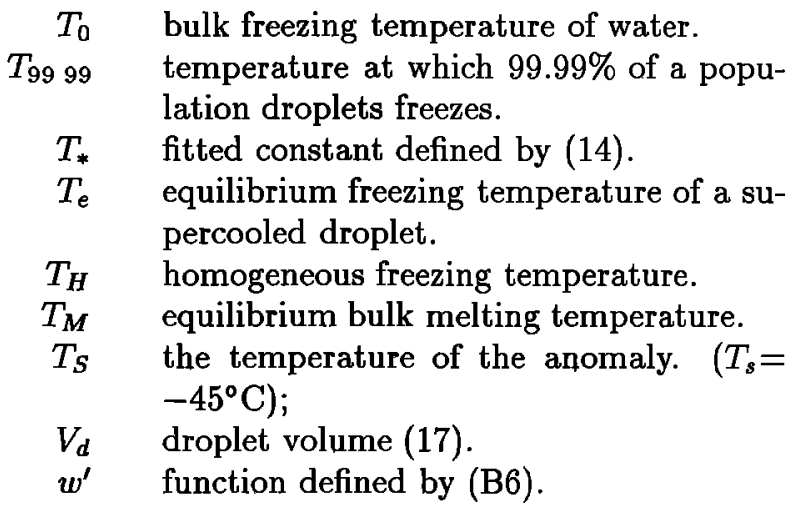

Acknowledgments. We are grateful to Marcia Baker for introducing us to Poole et al. [1994], to Jon Nelson for pointing out the homogeneous freezing measurements reported by MacKenzie [1977], to Marcia and Birger Bergersen for many helpful discussions, to two anonymous reviewers whoose comments improved the manuscript. This work is supported by grants from the Atmospheric Environment Service and the National Science and Engineering Research Council of Canada.

\section{References}

Angell, C. A., Supercooled water, in Water, A Comprehensive Treatise, edited by F. Franks, vol. 7, chap. 1, pp. 1-81, Plenum, New York, 1982.

Angell, C. A., M. Oguni, and W. J. Sichina, Heat capacity of water at extremes of supercooling and superheating, $J$. Phys. Chem., 86, 998, 1982.

Bartell, L. S., and J. Huang, Supercooling of water below the anomalous range near $226 \mathrm{~K}, J$. Phys. Chem., 98, 7455, 1994.

Butorin, G. T., and V. P. Skripov, Crystallization of supercooled water, Sov. Phys. Crystallogr., Engl. Transl., 17, 322, 1972.

Coriel, S. R., S. C. Hardy, and R. F. Skerka, A non-linear analysis of experiments on the morphological stability of ice cylinders freezing for aqueous solution, J. Cryst. Growth, 11, 53, 1971 .

Demott, P. J., and D. C. Rogers, Freezing nucleation rates of dilute solution droplets measured between $-30^{\circ} \mathrm{C}$ and $-40^{\circ} \mathrm{C}$ in laboratory simulations of natural clouds, J. At mos. Sci., 47, 1056, 1990.

Dufour, L., and R. Defay, Thermodynamics of Clouds, Academic, San Diego, Calif., 1963.

Eadie, W. J., A molecular theory of the homogeneous nucleation of ice from supercooled water, Ph.D. thesis, Univ. of Chicago, Chicago, Ill., 1971.

Floriano, M. A., and C. A. Angell, Surface tension and molar surface free energy and entropy of water to $-27.2^{\circ} \mathrm{C}, J$. Phys. Chem., 94, 4199, 1990.

Glasstone, S., K. J. Laidler, and H. Eyring, The Theory of Rate Processes, McGraw-Hill, New York, 1941.

Haar, L., J. S. Gallagher, and G. Kell, NBS/NRC Steam Tables, Hemisphere, Washington, D.C., 1984.

Hagen, D. E., R. J. Anderson, and J. L. Kassner, Homogeneous condensation-freeing nucleation rate measurements for small water droplets in an expansion cloud chamber, J. Atmos. Sci., 38, 1236, 1981.

Hardy, S. C., A grain boundary groove measurement of the surface tension between ice and water, Philos. Mag., 35, 471, 1977.

Hare, D. E., and C. M. Sorensen, The density of supercooled water, II, Bulk samples cooled to the homogeneous nucleation limit, J. Chem. Phys., 87, 4840, 1987. 
Harris, K. R., and L. A. Woolf, Pressure and temperature dependence of the self diffusion coefficient of water and oxygen-18 water, J. Chem. Soc. Faraday Trans. 1, 76, $377,1980$.

Heymsfield, A. J., and L. Miloshevich, Homogeneous ice nucleation of supercooled liquid water in orographic wave clouds, J. Atmos. Sci., 50, 2335, 1993.

Huang, J., and L. S. Bartell, Kinetics of homogeneous nucleation in the freezing of large water clusters, $J$. Phys. Chem., 99, 3924, 1995.

Jeffery, C. A., The thermodynamic behaviour of supercooled water: Results from a new equation of state, Master's thesis, Univ. of Br. Columbia, Vancouver, Br. Columbia, Can., 1996.

Jones, D. R. H., The measurement of solid-liquid interfacial energies from the shapes of grain-boundary grooves, Philos. Mag., 27, 569, 1973.

Kanno, H., R. J. Speedy, and C. A. Angell, Supercooling of water to $-92^{\circ} \mathrm{C}$ under pressure, Science, $189,880,1975$.

Ketcham, W. M., and P. Hobbs, Step growth on ice during freezing of pure water, Philos. Mag., 18, 659, 1969.

Krynicki, K., C. D. Green, and D. W. Sawyer, Pressure and temperature dependence of self-diffusion in water, $J$. Chem. Soc. Faraday Discuss. Chem. Soc., 66, 199, 1980.

Liu, X.-Y., The surface free energy of solid-fluid interfaces: An inhomogeneous cell model description, J. Chem. Phys., 98, 8154, 1993.

MacKenzie, A. P., Non-equilbrium freezing behaviour of aqueous systems, Phils. Trans. R. Soc. London, Ser. B, 278, 167, 1977.

McMullen, W. E., and D. W. Oxtoby, A theoretical study of the hard sphere fluid-solid interface, J. Chem. Phys., 88, 1967, 1988.

Melhem, G. A., R. Saini, and B. M. Goodwin, A modified Peng-Robinson equation of state, Fluid Phase Equilib., 47, 189, 1989.

Oxtoby, D. W., and P. R. Harrowell, The effect of density change on crystal growth rates from the melt, J. Chem. Phys., 96, 3834, 1992.

Poole, P. H., F. Sciortino, T. Grande, H. E. Stanley, and C. A. Angell, Effect of hydrogen bonds on the thermodynamic behavior of liquid water, Phys. Rev. Lett., 73, 1632, 1994.

Prielmeier, F. X., E. W. Lang, R. J. Speedy, and H.D. Lüdemann, The pressure dependence of self diffusion in supercooled light and heavy water, Ber. BunsenGes. Phys. Chem., 92, 1111, 1988.

Pruppacher, H. R., Self-diffusion coefficient of supercooled water, J. Chem. Phys., 56, 101, 1972.

Pruppacher, H. R., A new look at homogeneous ice nucleation in supercooled water drops, J. Atmos. Sci., 52, 1924, 1995.

Pruppacher, H. R., and J. D. Klett, Microphysics of Clouds and Precipitation, D. Reidel, Norwell, Mass., 1978.

Pruppacher, H. R., and J. D. Klett, Microphysics of Clouds and Precipitation, Kluwer Acad., Norwell, Mass., Boston, MA, 2nd ed., 1997.

Sassen, K., and G. C. Dodd, Homogeneous nucleation rate for highly supercooled cirrus cloud droplets, J. Atmos. Sci., 45, 1357, 1988.

Song, Y., and E. Mason, Statistical-mechanical theory of a new analytical equation of state, J. Chem. Phys., 91, $7840,1989$.

Song, Y., and E. A. Mason, Analytical equation of state for molecular fluids: Kihara model for rodlike molecules, Phys. Rev. A, 42, 4743, 1990a.

Song, Y., and E. A. Mason, Analytical equation of state for molecular fluids: Comparison with experimental data, Phys. Rev. A, 42, 4749, 1990b.

Speedy, R. J., Limiting forms of the thermodynamic divergences at the conjectured stability limits in superheated and supercooled water, J. Phys. Chem., 86, 3002, 1982a.

Speedy, R. J., Stability-limit conjecture: An interpretation of the properties of water, J. Phys. Chem., 86, 982, 1982b.

Speedy, R. J., Two waters and no ice please, Nature, 380, 289,1996

Speedy, R. J., and C. A. Angell, Isothermal compressibility of supercooled water and evidence for a thermodynamic singularity at $-45^{\circ} \mathrm{C}, J$. Chem. Phys., 65, 851, 1976.

Tanaka, H., A self-consistent phase diagram for supercooled water, Nature, 380, 328, 1996.

Turnbull, D., Formation of crystal nuclei in liquid metals, $J$. Appl. Phys., 21, 1022, 1950.

Wagner, W., A. Saul, and A. Pruss, International equations for the pressure along the melting and along the sublimation curve of ordinary water substance, J. Phys. Chem. Ref. Data, 23(3), 515, 1994.

Wang, J. H., Tracer-diffusion in liquids, III: The selfdiffusion of chloride ion in aqueous sodium solutions, $J$. Am. Chem. Soc., 74, 1612, 1952.

Wood, G. R., and A. G. Walton, Homogeneous nucleation kinetics of ice from water, J. Applied Phys., 41, 3027, 1970.

Woolf, L. A., Tracer diffusion of tritiated water (THO) in ordinary water $\left(\mathrm{H}_{2} \mathrm{O}\right)$ under pressure, J. Chem. Soc. Faraday Trans. 1, 71, 784, 1975.

Xans, P., and G. Barnaud, Thermodynamique, C. R. Acad. Sc. Ser. B, 280, 25, 1975.

Xie, Y., K. F. Ludwig, Jr., and G. Morales, Noncritical behavior of density fluctuations in supercooled water, Phys. Rev. Lett., 71, 2050, 1993.

P.H. Austin and C.A. Jeffery, Atmospheric Sciences Programme, \#217 Geography, 1984 West Mall, University of British Columbia, Vancouver, B.C. V6T1Z2 Canada. (e-mail: phil@geog.ubc.ca; cjeff@geog.ubc.ca)

(Received March 3, 1997; revised July 28, 1997; accepted July 31, 1997.) 